\title{
ACIDENTES OFÍDICOS CAUSADOS POR Bothrops moojeni: CORRELAÇÃO DO QUADRO CLÍNICO COM O TAMANHO DA SERPENTE
}

\author{
Joáo Aris Kolyoumbjian (1) \& Cristina Polizelli (2)
}

\begin{abstract}
RESUMO
Durante um periodo de 19 meses (março 1986 a setembro 1987) foram estudados 22 casos de acidentes ofídicos causados por Bothrops moojeni na regiāo de São José do Rio Preto. São Paulo, nos quais o tamanho da serpente foi sistematicamente medido. Foram constituidos dois grupos de pacientes de acordo com o tamanho da serpente: grupo I -.- 9 casos de serpentes pequenas $(30$ a $53 \mathrm{~cm}$ ) e grupo II 13 casos de serpentes grandes $(80$ a $147 \mathrm{~cm})$. Os resultados mostraram: 1. efeitos locais iniciais - dor e edema - mais leves no grupo I; 2 . tempo de coagulaçāo prolongado/incoagulável levemente mais frequente no grupo I: 3 . complicaçóes locais - necrose, infeç̧áo e síndrome compartimental - exclusivamente, e em mais da metade dos casos do grupo II, apesar da terapia com antiveneno ter sido mais rápida e em doses maiores nesse grupo. Conclui-se que as serpentes Bothrops moojeni maiores apresentam grande incremento nas suas açoes locais - edema, necrose e infecçáo secundária - e leve perda em sua açāo coagulante.
\end{abstract}

UNITEKMOS: Acidente ofidico humano: Acidente botrópico humano: Bothrops moojeni.

\section{INTRODUÇÃO}

Os acidentes ofídicos botrópicos na regiāo de São José do Rio Preto, São Paulo, são em sua grande maioria causados por Bothrops moojeni $^{3}$. Em trabalho anterior pudemos caracteri zar esse tipo específico de acidente com relaçāo à epidemiologia, quadro clínico, diagnostico, tratamento e complicaçōes, salientando as prin cipais diferenças com relaçăo a acidentes botró picos descritos para outras especies ${ }^{5}$. Mais re centemente, baseando-se em estudos experi mentais já bem conhecidos de venenos ofídicos em animais de laboratório ${ }^{11}$, tem sido descrito diferenças no quadro clínico dos pacientes acidentados por serpentes jovens ou adultas a $^{2 .}$. 8 , 9. 10

O objetivo do presente trabalho é relatar e comparar os achados clínicos dos acidentes ofí-

Trabalho realizado no Hospital de Base da Fundaça Faculdade Regional de Medicina de Sảo José do Rio Preto. Sảo Paulo, Brasil.

(1) Professor Assistente do Departamento de Medicina Integrada e Chefe da Disciplina de Neurologia do Hospital de Base da Fundaçāo Faculdade Regional de Medicina de Sao Jose do Rio Preto. Sāo Paulo. Brasil.

(2) Medica Chefe da Unidade de Terapia Intensiva do Hospital de Base da Fundacao Faculdade Regional de Medicina de Sáo José do Rio Preto. Sáo Paulo. Brasil

Endereço para correspondència: Dr. Joào Aris Kouyoumdjian. Av. Bady Bassitt, 3896. CEP 15015 Sảo José do Rio Preto. Sâo Paulo. Brasil 
KOUYOUMDJIAN, J. A. \& POLIZELLI, C. - Acidentes ofidicos causados por Bothrops moojeni: correlaçāo do quadro clínico com o tamanho da serpente. Rev. Inst. Med. trop. Sáo Paulo, 31(2): 84 90, 1989.

dicos causados por Bothrops moojeni pequenas e grandes.

\section{MATERIAL E MÉTODOS}

Todos os casos de acidentes ofídicos que de ram entrada no Hospital de Base da Fundação Faculdade Regional de Medicina de Sāo José do Rio Preto, Sāo Paulo, com identificaçảo e medida da serpente agressora, no periodo de março de 1986 a setembro de 1987 , foram estudados de maneira prospectiva. Os casos foram cadastrados em um protocolo especialmente feito para o estudo de acidentes ofídicos na regiảo; todos foram atendidos e classificados apenas pe los autores.

Foram selecionados para o presente traba lho, 22 casos em que a serpente agressora foi iden tificada como Bothrops moojeni; por motivos práticos, a medida do comprimento do animal quase sempre foi realizada após administração do antiveneno. A identificaçāo foi feita no Depar tamento de Zoologia, IBILCE/UNESP. São José do Rio Preto, Sảo Paulo.

Foram constituidos dois grupos de aciden tados: Grupo I, formado por 9 casos de acidentes causados por serpentes pequenas ( 30 a 53 , média de $36 \mathrm{~cm}$ ) e Grupo II, formado por 13 casos de acidentes causados por serpentes grandes 180 a 147, média de $104,3 \mathrm{~cm}$.

TABELA

Acidentes por Bothrops moojeni : Grupo I - pequenas e Grupo II - grandes.

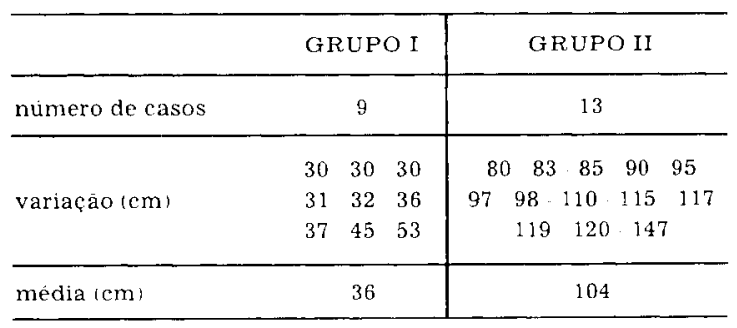

Os itens enfatizados foram: segmento corpó reo atingido, quadro clínico, tempo de coagu lação, soroterapia, complicações e tempo de in ternaçāo

O exame do tempo de coagulação (TC) foi feito colocando-se $2 \mathrm{ml}$ de sangue em tubo de ensaio, deixando-se em banho-maria a $37^{\prime \prime} \mathrm{C}$ e a cada minuto verificando-se se houve coagulaçào (normal até 10 minutos, prolongado de 11 a 30 minutos e incoagulável quando maior que $30 \mathrm{mi}$ nutosi.

Todos os antivenenos utilizados foram do Instituto Butantan: soro antibotrópico $(10 \mathrm{ml}$ neutralizando $25 \mathrm{mg}$ de veneno botrópicol ou na falta desse, soro-anti-ofídico $110 \mathrm{ml}$ neutralizando $20 \mathrm{mg}$ de veneno botrópicol. A administração do antiveneno foi sempre realizada por via endovenosa.

As complicaçōes clínicas e/ou cirúrgicas fo ram tratadas no mesmo hospital em setor com petente. Em alguns casos não foi possivel o se. guimento a longo prazo para a avaliaçāo de complicaçōes tardias do quadro clínico.

Os métodos estatísticos utilizados foram: média, desvio padrāo e test $t$-Student com grau de liberdade $n-1$ e significância 0,01 e $0,05 \mathrm{cp}$ $>0,05$ não significante, $p \leqslant 0,05$ provavelmente significante e $p \leqslant 0,01$ altamente significante).

\section{RESULTADOS}

\section{Segmento Corpóreo Atingido}

A tabela 2 mostra a distribuição dos diferentes segmentos corpóreos atingidos nos dois grupos. Nảo houve diferença significativa entre os dois grupos, apesar da diferença de tamanho dos animais.

TABELA 2

Acidentes por Bothrops moojeni: segmento atingido nos 2 grupos

GRUPO I GRUPO II

\begin{tabular}{ccc}
\hline $\begin{array}{c}\text { Pé } \\
\text { Tornozelo } \\
\text { Terço inferior perna }\end{array}$ & $7(77,8 \%)$ & $10(77 \%)$ \\
\hline Mão & $1(11,1 \%)$ & $3(23 \%)$ \\
\hline Joelho & $1(11,1 \%)$ & - \\
\hline
\end{tabular}

\section{Quadro Clínico}

A comparação foi feita entre os itens anormais da avaliaçāo clínica e tem po de coagulação 
KOUYOUMDJIAN, J. A. \& POLIZELLI, C. - Acidentes ofídicos causados por Bothrops moojeni: correlaçáo do quadro clínico com o tamanho da serpente. Rev. Inst. Med. trop. Sáo Paulo, 31(2): 84 90, 1989

iniciais. O TC é abordado dentro de quadro clíni co pois é parte integrante do diagnóstico de acidentes ofidicos. Um paciente do grupo I foi excluido da comparaçāo devido à ausência de qualquer anormalidade clínico-laboratorial na admissão (considerado como acidente sem envene namento). Os efeitos locais imediatos, ou seja, de aparecimento nos primeiros 30 minutos do acidente, foram classificados de acordo com a extensāo do edema: leve $\left(\mathrm{L}_{\mathrm{L}} /+\right)$ com edema peripicada, moderado $(\mathrm{M} /++$ ) com edema regional e grave $(G /+++$ ) com edema em todo membro. O TC imediato e seqüencial foi feito segundo método já descrito e as hemorragias sistêmicas foram constatadas pelo exame clínico. Os efeitos locais apareceram em todos os casos do grupo I e grupo II; a comparação revelou diferença significativa nos efeitos locais leves, que ocorreram em proporção maior no grupo I; não ocorreram casos graves no grupo I. O TC prolongado ocor reu em $75 \%$ do grupo I e em $61,6 \%$ do grupo II, resultado sem diferença significativa. Hemorragia sistêmica (hemorragia digestiva alta) ocorreu em apenas 1 caso $(7,7 \%)$ do grupo II e em nenhum caso do grupo I. A tabela 3 compara todos os dados obtidos.

TABELA 3

Acidentes por Bothrops moojeni: quadro clinico tempo de coagulaçāo nos dois grupos.

\begin{tabular}{cccc}
\hline & & $\begin{array}{c}\text { GRUPO I } \\
(8 \%)\end{array}$ & $\begin{array}{c}\text { GRUPO II } \\
(13 \% 13)\end{array}$ \\
\hline \multirow{2}{*}{ Efeitos Locais } & Leve & $4(50 \%)$ & $1(7.7 \%)$ \\
\cline { 2 - 4 } & Moderado & $4(50 \%)$ & $9(69.3 \%)$ \\
\cline { 2 - 4 } TC & Grave & nenhum & $3(23 \%)$ \\
\hline Normal & $2(25 \%)$ & $5(38,4 \%)$ \\
\cline { 2 - 4 } & Prolongado & $6(75 \%)$ & $8(61.6 \%)$ \\
\hline \multirow{2}{*}{ Hemorragia Sistémica } & & nenhum & $1(7.7 \%)$ \\
\hline
\end{tabular}

\section{Soroterapia}

Foram comparados todos os casos dos dois grupos em que a soroterapia foi indicada. A tabela 4 mostra os valores médios comparativos dos sub-itens estudados entre os dois grupos. Nāo houve indicação de soroterapia para um caso do grupo I.

\subsection{Tempo para Soroterapia Inicial}

Tempo médio decorrido entre o acidente e a administração do antiveneno
4 horas e 37 minutos \pm 2 horas e 41 minutos (grupo I) 3 horas e 41 minutos \pm 1 hora e 17 minutos (grupo II). A comparação mostra resultado provavelmente significativo $(p \leqslant 0,05$ e $p>0,01)$. com administração mais rapida de antıvene no no grupo II.

\subsection{Quantidade Inicial de Antiveneno}

Quantidade inicial média de antive neno botrópico administrado, calculado em miligramas de veneno a serem neu tralizados: $137 \pm 37$ (grupo I) e $166 \pm$ 58 (grupo II). A comparação das médias mostra ausência de significância ( $p$ > 0,05 ).

\subsection{Tempo para Soroterapia Adicional}

Antiveneno botrópico adicional foi indicado em 2 casos $(25 \%)$ do grupo I e em 5 casos $(38,4 \%)$ do grupo II, na persistência de TC prolongado após 8-12 horas ou na evidente progressāo dos efeitos locais para maior gravidade. O tempo médio foi de 7 horas e 30 minutos \pm 4 horas e 30 minutos (grupo I) e de 17 horas e 30 minutos \pm 10 horas e 44 minutos (grupo II). A comparação das médias mostra ausência de significância $(p>0,05)$.

\subsection{Quantidade Adicional de Antiveneno}

Quantidade adicional média de antiveneno botrópico administrado, calcula do em miligramas de veneno a serem neutralizados: 50 (grupo I) e $90 \pm 25$ (grupo II). A comparaçāo das médias mostra ausência de significância $(\mathrm{p}>0,05)$.

\subsection{Quantidade Total de Antiveneno}

Quantidade total média de antive neno botrópico administrado (inicial e adicional), calculado em miligramas de veneno a serem neutralizados: $150 \pm 46$ (grupo I) e $201 \pm 72$ (grupo II). A compa raçāo das médias mostra ausência de significância $(\mathbf{p}>0,05)$.

\subsection{Tempo de Normalização do TC após So- roterapia}

A média desse tempo nos casos em que o TC inicial estava prolongado foi de 14 horas e 50 minutos \pm 5 horas e 
KOUYOUMDJIAN, J. A. \& POLIZELLI, C. - Acidentes ofidicos calisados por Bothrops moojeni: correlaçáo do quadro clínico com o tamanho da serpente. Rev. Inst. Med. trop. Sảo Paulo, 31/2):8490, 1989.

20 minutos (grupo I) e de 16 horas e 30 minutos \pm 10 horas e 50 minutos (grupo
II). A comparação das médias mostra au sência de significância $(p>0.05)$.

TABELA

Acidentes por Bothrops moojeni: terapia com antiveneno - tempo, concentraçoes neutralizantes e normalizaçāo do TC nos 2 grupos

\begin{tabular}{|c|c|c|c|c|}
\hline & & GRUPOI $(89)$ & GRUPO II ( $13: 13$ ) & SIGNIFICÁNCIA \\
\hline \multirow{2}{*}{$\begin{array}{l}\text { Antiveneno Inicial } \\
\qquad(21 / 21)\end{array}$} & Tempo & $4 \mathrm{hs} 37 \mathrm{~min} \pm 2 \mathrm{hs} 4 \mathrm{~min}$ & 3hs4lmin $-1 \mathrm{~h} 17 \mathrm{~min}$ & $p \leqslant 0,05: p>0,01$ \\
\hline & $\operatorname{Dose}^{f}$ & $137 \pm 37$ & $166+58$ & $p>0,05$ \\
\hline \multirow{2}{*}{$\begin{array}{l}\text { Antiveneno Adicional } \\
\qquad(721)\end{array}$} & Tempo & $7 \mathrm{hs} 30 \mathrm{~min} \pm 4 \mathrm{hs} 30 \mathrm{~min}$ & $17 \mathrm{hs} 30 \mathrm{~min}-10 \mathrm{hs} 44 \mathrm{~min}$ & $\mathrm{p} \rightleftharpoons 0,05$ \\
\hline & Dose $*$ & $50 \pm 0$ & $90 \div 25$ & $p>0,05$ \\
\hline $\begin{array}{l}\text { Antiveneno Total } \\
\qquad(2121)\end{array}$ & Dose ${ }^{*}$ & $150=46$ & $201 \pm 72$ & $p>0,05$ \\
\hline $\begin{array}{c}\text { Normalizacáo do TC } \\
(14 / 21)\end{array}$ & Tempo & $14 \mathrm{hs} 50 \mathrm{~min}=5 \mathrm{hs} 20 \mathrm{~min}$ & $16 \mathrm{hs} 30 \mathrm{~min}-10 \mathrm{hs} 50 \mathrm{~min}$ & $\mathrm{p}>0,05$ \\
\hline
\end{tabular}

(*) Quantidade de veneno neutralizado em miligramas.

\section{Complicaçóes}

As complicaçōes ocuriroram em 7 Lasos $(53,8 \%)$ do grupo II e em nenk. caso do grupo I, sendo tal diferença muito significativa para o grupo II. Não ocorreram casos de choque, coagulação intravascular disseminada ou insuficiência renal, apesar da ocorrência de um óbito de causa nào determinada. Todas as complicaçōes ocorreram em pacientes que tiveram avalia ção inicial moderado ou grave e em alguns pa cientes elas se somaram. A tabela 5 mostra a distribuição das complicaçōes

\section{1. Óbito}

Houve um óbito $(7,6 \%)$ no grupo II no terceiro dia de evolução; o caso foi considerado como grave na avaliaçâo inicial com sindrome compartimental e fascio tomia precoce; não ocorreram óbitos no grupo I.

\subsection{Síndrome Compartimental}

Definida como edema volumoso do membro atingido com prejuízo à circula çāo: 2 casos (15,3\%) no grupo II com ne cessidade de fasciotomia precoce e ne nhum caso no grupo I.

\subsection{Necrose}

Definida como perda tecidual (pele subcutâneo / músculo) ou de todo seg mento atingido (amputação): 2 casos
$(15,3 \%)$ no grupo II e nenhum no grupo I.

\subsection{Infecção}

Definida como secreçāo purulenta difusa ou septada (abscesso): 4 casos $(30,7 \%)$ no grupo II e nenhum no grupo I. A comparação revelou diferença signi ficativa desse item para os casos do gru po II.

TABELA 5

Acidentes por Bothrops moojeni: complicações nos 2 grupos.

\begin{tabular}{ccc}
\hline & $\begin{array}{c}\text { GRUPO I } \\
(8 / 9)\end{array}$ & $\begin{array}{c}\text { GRUPO II } \\
(13 / 13)\end{array}$ \\
\hline Óbito & nenhum & $1(7.6 \%)$ \\
\hline Necrose & nenhum & $2(15,3 \%)$ \\
\hline Infecção & nenhum & $4(30,7 \%)$ \\
\hline $\begin{array}{c}\text { Sindrome } \\
\text { Compartimental }\end{array}$ & nenhum & $2(15,3 \%)$ \\
\hline Total & nenhum & $7(53,8 \%)$ \\
\hline
\end{tabular}

A correlação da gravidade inicial ( $L-M$ - G) com diversas variáveis indicadas na tabela 6 , mostra que todos os casos graves foram picados por serpentes grandes, evoluindo para com 
KOUYOUMdjiAN. J. A. \& POLIZELLI, C. - Acidentes ofídicos causados por Bothrops moojeni: correlaçáo do quadro clinico com o tamanho da serpente. Rev. Inst. Med. trop. São Paulo, 31(2): 84-90, 1989.

plicaçóes. Todos os casos de avaliação inicial leve tiveram incoagulabilidade sangüinea, sendo a maioria dos acidentes causados por serpentes pequenas. Não houve antecedentes mórbidos importantes tais como diabetes mellitus, vascu- lopatias definidas, insuficiência tenal grave ou doenças hematológicas entre os três grupos de gravidade. A idade provavelmente representou fator agravante, porém a amostragem é reduzida, nāo permitindo conclusāo definitiva.

TABELA 6

Correlaçāo de gravidade inicial do acidente com incidencia, tamanho da serpente, idade, sexo, incoagulabilidade sangünea e complicaçòes

\begin{tabular}{|c|c|c|c|c|c|c|c|}
\hline & \multirow{2}{*}{$\begin{array}{c}\text { N: CASOS } \\
\text { (21) }\end{array}$} & \multicolumn{2}{|c|}{ TAMANHO } & \multirow{2}{*}{$\begin{array}{l}\text { IDADE } \\
\text { (ANOS) }\end{array}$} & \multirow{2}{*}{$\begin{array}{l}\text { SEXO } \\
(\mathrm{M}: \mathrm{F})\end{array}$} & \multirow{2}{*}{$\begin{array}{c}\text { INCOAGULABILIDADE } \\
\text { SANGUINEA }\end{array}$} & \multirow{2}{*}{ COMPLICAÇOESS } \\
\hline & & $\begin{array}{l}\text { PEQUENO } \\
(30-53 \mathrm{~cm})\end{array}$ & $\begin{array}{c}\text { GRANDE } \\
(80-147 \mathrm{~cm})\end{array}$ & & & & \\
\hline Leve & 5 & 4 & 1 & $\begin{array}{r}645 \\
(23.6)\end{array}$ & $4: 1$ & $5(100 \%)$ & nenhum \\
\hline Moderado & 13 & 4 & 9 & $\begin{array}{c}155 \\
(32,4)\end{array}$ & $10: 3$ & $7(53.8 \%)$ & $4130.7 \%$ \\
\hline Grave & 3 & nenhum & 3 & $\begin{array}{l}2269 \\
152.31\end{array}$ & $3: 0$ & $2\left(33,3 c_{k}\right)$ & $3(100 \%)$ \\
\hline
\end{tabular}

\section{Tempo de Internação}

O tempo médio total de internação é mos trado na tabela $7: 2,7 \pm 0,8$ dias para o grupo Ie $12,7 \pm 12,3$ dias para o grupo II. A comparação revelou resultado altamente significante $(p \leqslant$ 0,01 ).

TABELA 7

Acidentes por Bothrops moojeni: tempo de internaçào nos 2 grupos

\begin{tabular}{cccc}
\hline & Grupo I (8:9) & Grupo II 11313 ) Significancia \\
\hline $\begin{array}{c}\text { Tempo Médio } \\
\text { de } \\
\text { internaçåo }\end{array}$ & $2.7 \pm 0.8$ dias & $12,7 \pm 12.3$ dias & $p=0.01$ \\
\hline
\end{tabular}

\section{DISCUSSÃO}

Os acidentes ofidicos botrópicos na regiào de Sāo José do Rio Preto, Sāo Paulo, são em sua grande maioria causados por Bothrops moojeni, cujo veneno origina ações necróticas locais mais severas e maior percentagem de distúrbios de coagulação, com tempo de coagulaçāo prolongado em aproximadamente $75 \%$ dos ca$\operatorname{sos}^{3.5}$.

A correlaçāo do quadro clínico com tama nho da serpente Bothrops moojeni ainda năo foi determinado em nosso meio, apesar do nosso pequeno relato anterior ${ }^{4}$. Já de há muito sabe-se que as serpentes Bothrops jararaca filhotes apresentam uma fraçāo coagulante mais forte e uma fraçāo proteolitica mais fraca que as adultas, como comprovam trabalhos clínicos recen tes 1.2 .6 .8 .8 .10 .

Na nossa casuística não houve diferença significativa entre os locais da picada nos 2 grupos, porém em grandes amostragens já se comprovou que pelo tamanho do animal, obviamente o bote será mais ou menos longo, podendo atingir mais pé e tornozelo em serpentes pequenas e pé, tornozelo e perna em proporçóes parecidas para as serpentes maiores ${ }^{10}$. O quadro clínico revelou que no grupo de serpentes pequenas houve predominância de edema inicial leve, menor percentagem de moderado e nenhum caso grave quando comparado ao grupo de serpentes maiores. O tempo de coagulaçāo prolongado/incoagulável foi mais freqüente no grupo de serpentes pe quenas sem diferença significativa. As hemorragias sistêmicas foram raras, ocorrendo em apenas um caso do grupo maior que inclusive apresentava TC normal, mostrando que a desfibrino genação não representou a causa primária das hemorragias ${ }^{7}$; os fatores hemorrágicos provavel mente foram neutralizados com eficiência pelo antiveneno botrópico.

O tratamento inicial com antiveneno foi mais rápido (estatisticamente significante) e em 
KOUYOLMDJIAN, J. A. \& POLIZELLI, C. - Acidentes ofídicos causados por Bothrops moojeni: correlaçăo do quadro clínico com o tamanho da serpente. Rev. Inst. Med. trop. Sáo Paulo, 31(2): $8490,1989$.

concentraçōes neutralizantes mais elevadas no grupo de serpentes maiores; antiveneno adicional também foi administrado em doses maiores nesse grupo. A quantidade total de antiveneno administrado foi, portanto, mais elevada no gru po de serpentes maiores. O tempo de norma lizaçāo do TC foi parecido nos dois grupos, mostrando boa neutralizaçāo da fraçāo coagulante e dos fatores hemorrágicos do veneno mesmo com antiveneno em concentraçōes neutralizantes mais baixas no grupo de serpentes pequenas. As complicações ocorreram exclusivamente e em mais da metade dos casos do grupo de serpentes maiores, diferença altamente significativa; isoladamente, a infecçāo foi a complicação mais comum. O tempo de internaçāo foi muito maior nesse grupo, com diferença altamente significante.

Os nossos resultados revelam que a maior diferença clínica entre os pacientes picados por serpentes Bothrops moojeni pequenas e grandes está no grande incremento das açōes locais edema, necrose e infecção secundária - nas últi mas, devido provavelmente à maior quantidade de veneno inoculado e à maior açāo proteolítica. As anormalidades da coagulaçāo sangüínea, traduzidas pela avaliaçāo do TC, nāo mostraram diferenças significativas, apesar de ocorrerem mais em serpentes pequenas. Apesar da administração de antiveneno mais rápido e em doses maiores no grupo de serpentes grandes, as complicaçōes ocorreram somente nesse grupo. Os acidentes causados por Bothrops moojeni gran des com avaliaçăo inicial moderado/grave apre sentam enorme possibilidade de complicações locais evolutivas, mesmo pós terapia com antiveneno em doses adequadas. Desde que esse fator prognóstico exista, estudos subseqüentes são necessários para se avaliar a possibilidade de terapia cirúrgica precoce, pós normalização do TC.

\section{SUMMARY}

\section{SNAKE BITE ACCIDENTS CAUSED BY Bothrops moojeni: CLINICAL PICTURE RELATED TO THE ANIMAL LENGHT}

During a period of 19 months (March 1986 - September 1987), 22 cases of snake bites cau sed by Bothrops moojeni in Sāo José do Rio Preto, Sāo Paulo (South-east Brazil) were stu died. The animals have their lenght measured and two groups were constituted: group I -9 cases with lenght between 30 and $53 \mathrm{~cm}$ and group II -13 cases with lenght between 80 and $147 \mathrm{~cm}$. Our results revealed: 1 . the local effects - pain and swelling - were frequently mild in group I; 2 - non-clotting blood (bed-side test) occured in two groups, a little more frequently in group $\mathrm{I}$; 3 . local complications - necrosis, compartment syndrome and infection - occured in more then a half and exclusively in group II, in spite of larger and faster antivenom therapy. We have concluded that larger Bothrops moojeni produced great increment in swelling, necrosis and infection and mild decrement in coagulation action.

\section{AGRADECIMENTO}

Professor-Doutor Valdir Antonio Taddei, Chefe do Departamento de Zoologia, IBILCE/UNESP - São José do Rio Preto, São Paulo, pela identificaçảo das serpentes.

\section{REFERENCIAS BIBLIOGRAFICAS}

1. BELLUOMINI, H. E. -Conhecimentos sobre as serpentes brasileiras e medidas de prevençáo de acidentes. Rev. bras. Saúde ocup., 12: 82.96, 1984.

2. FRANÇA, F, O. S. \& CARDOSO, J, L. C. - Estudo retros pectivo da evoluçào de acidentes botrópicos. Rev. Soc. bras. Med. trop., 20 (supl.): 56, 1987.

3. KOUYOUMDJIAN, J. A.; ABDELNUR, C. P.; FARES, G F.; FONSECA, M. G. \& KOUYOUMDJIAN, N. C. V. Envenenamentos ofidicos na regiāo de Sāo José do Rio Preto. Estudo prospectivo de 49 casos. Rev. Soc. bras. Med. trop., 19 (supl.): 27, 1986.

4. KOUYOUMDJIAN, J. A.; POLIZELLI, C.; FONSECA, M G.; FARES, G. F.; NAKAOSKI, S. C. B.; ANANIAS, M. P. \& KOUYOUMDJIAN, N. C. V. - Acidentes ofidicos causados por Bothrops moojeni na regiāo de Sáo José do Rio Preto: correlaçăo do quadro clínico com o tamanho da serpente. Rev. Soc. bras. Med. trop., 20 isupl.): 53-54, 1987.

5. KOUYOUMDJIAN, J. A.; FONSECA, M. G.; POLIZELLI, C.; FARES, G. F.; NAKAOSKI, S. C. B.; ANANIAS, M. P. \& KOUYOUMDJIAN, N. C. V. - Acidentes ofidicos causados por Bothrops moojeni na regiāo de Sāo José do Rio Preto: estudo de 27 casos. Rev. Soc. bras. Med. trop., 20 (supl.1: 53, 1987.

6. LOPES, P. F. A.; MARQUES, M. M. A.; CAMPOS, J. A.; CARDOSO, J. L. C.; KOUYOUMDJIAN, J. A.; DOURA DO, H. V. \& AMARAL, C. F. S. - Manual de diagnóstico e tratamento de acidentes ofídicos. Brasilia, Ministério 
KOUYOUMDJIAN. J. A. \& POLIZELLI. C. - Acidentes ofidicos causados por Bothrops moojeni: correlaçảo do quadro elinico com o tamanho da serpente. Rev. Inst. Med. trop. Sáo Paulo, 31(2): 84-90, 1989.

da Saude, 1987. Serio A: Normas e Manuais Teenicos, Il 331 .

7. FEID. H A. \& THEAKSTON, R, D. G. - The manago ment of snake bita. Bull. Wld. H1th. Orr., 61:885 895,1983

8. RIBEIRO L A \& JORGE, M. T. ... Alteracios do tempo dt: coagulaço sanguinea om pacientes picacios por Bo. throps jararaca filhotec adulta. Rev. Soc. bras. Med. trop. 18 isupl.1:27 28,1985

9. RIBEIRO. L. A. JORGE, M. T. \& CARDOSO, J. L. C Fatores prognostioos em acidentes por serpentes do genero Bothrops. Estudo de 307 casos. Rev. Soc. bras Med. trop., 18 isupl.i: 28.1985

10. RIBEIRO. L. A. \& JORGE. M. T. Avaliaçào por Bothrops jararaca adulta e filhote: diferences clinicas. epide miológicas e na capacidade de alterar a coagulaçāo san guinea. Rev. Soc. bras. Med. trop., 20 (supl.): 54, 1987.

11. ROSENFELD, G.: HAMPE, O. G. \& KELEN, E. M. A - Coagulant and fibrinolytic activity of animal venoms determination of coagulant and fibrinolytic index of diffe rent species. Mem. Inst. Butantan, 29: 143163,1959

Recebido para publicaça em $12.09: 1988$ 